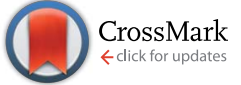

Cite this: J. Mater. Chem. A, 2016, 4, 2246

Received 29th November 2015 Accepted 6th January 2016

DOI: 10.1039/c5ta09709f

www.rsc.org/MaterialsA

\section{Polarization induced dynamic photoluminescence in carbon quantum dot-based ionic fluid $\dagger$}

\author{
Lopamudra Bhattacharjee, ${ }^{a}$ Kallol Mohanta, ${ }^{a}$ Kaushik Pal, ${ }^{\mathrm{b}}$ Apurba L. Koner ${ }^{\mathrm{b}}$ \\ and Rama Ranjan Bhattacharjee*a
}

\begin{abstract}
We have recently reported the synthesis of water-dispersible, polymer-passivated and redox-active carbon quantum dots (CQDs). The CQDs were converted into a solvent-less conductive fluid through a step-wise surface modification technique. The material has a core-corona-canopy structure with CQD as the core, passivating-polymer as the corona and polyetheramine (Jeffamine $®$ ) as the canopy. These materials are unique in characteristics and are designated as nano-ionic materials (NIMs). Structure and properties of CQD-NIMs were determined by dynamic light scattering, thermogravimetry, differential scanning calorimetry, photoluminescence $(\mathrm{PL})$ and cyclic voltammetry $(\mathrm{CV})$. Dynamic changes in extrinsic $\mathrm{PL}$ maxima $\left(\lambda_{\mathrm{em}}\right)$ of the CQDs were observed during and after CV. Such fluctuations in $\lambda_{\mathrm{em}}$ helped to understand the sequential ordering and disordering of the Jeffamine ${ }^{\circledR}$ canopy on the CQD surface during polarization during CV. This phenomenon enables us to understand molecular canopy dynamics in NIMs and further showcases redox-active CQDs as a sustainable material for future electrochemical applications.
\end{abstract}

\section{Introduction}

Functional nanomaterials are currently at the forefront of material research. Surface passivation and functionalization of nanomaterials enable control over their physicochemical properties and enhance their multidirectional applicability. The surface structure in these materials plays a vital role in predicting the way these systems function. One such interesting structure is the core-corona-canopy design in fluid-like nanoionic materials (NIMs). First introduced by Giannelis and coworkers, these functional nanomaterials show interesting rheological properties. ${ }^{1,2}$ The literature suggests that the fluidic properties are tunable with variations in the type of core-corona and nature of the chosen canopy. ${ }^{3-5}$ We had earlier reported surface-modification of gold-nanorod (GNR) corona with a Jeffamine® canopy through electrostatic self-assembly, that resulted in a plasmonic fluid. ${ }^{3}$ Shear-induced dynamic changes in the plasmonic characteristics of GNRs were monitored and attributed to the Jeffamine® canopy induced slow relaxation/ rearrangement of the GNR assemblies. ${ }^{3}$ Dynamics of the canopy determine the fluidic property in NIMs and can be probed using

${ }^{a} P S G$ Institute of Advanced Studies, Coimbatore, Tamil Nadu, 641004, India. E-mail: ramaranjanb89@gmail.com; Fax: +91-422 257-3833; Tel: +91-422-434-4000

${ }^{b}$ Department of Chemistry, Indian Institute of Science Education \& Research Bhopal, Bhopal By Pass Road, Bhauri, Bhopal, 462066, India. Fax: +91-755-669-2392; Tel: +91-755-669-2376

$\dagger$ Electronic supplementary information (ESI) available: Life-time measurements, solubility results, CV of pure Jeffamine ${ }^{\circledR}$ and $\mathrm{CV}$ of 10 consecutive cycles for CQD-NIMs. See DOI: 10.1039/c5ta09709f rheological, neutron scattering and NMR techniques. ${ }^{1-6}$ The results obtained from NMR and rheological studies indicated that the liquid-like behavior in NIMs is due to the rapid exchange of the canopy between the ionically-modified nanoparticles used as core materials. ${ }^{6}$ Among various core materials reported so far, a few carbon-based core materials have been used for NIM studies. Giannelis et al. have reported the synthesis of NIMs by neutralizing fully protonated fullerol with an amine terminated polyetheramine that exhibited complex viscoelastic behavior. ${ }^{4}$ Graphene oxide quantum dots have been modified into a fluid by Zhang et al. that exhibited excellent solubility and amphiphilicity. ${ }^{5}$

Carbon quantum dots (CQDs) are a new class of interesting carbon-based nanostructures that can be produced from various carbon resources. ${ }^{7-12}$ The main challenge for chemists is to tune the structure of the CQDs by controlling the density of $\mathrm{sp}^{2}$ islands in the $\mathrm{sp}^{3}$ matrix. ${ }^{12,13}$ CQDs have consistently gained interest due to their non-toxic nature, unique photoluminescence ${ }^{12-14}$ (PL) and conducting properties. ${ }^{15,16}$ Wen et al. reported that the PL of CQDs consists of two spectral bands. These bands are termed intrinsic and extrinsic bands and correspond to band gap and surface trap states, respectively. ${ }^{\mathbf{1 2 - 1 4}}$ The bands can be tuned by tuning the size of $\mathrm{sp}^{2}$ nano-domains and the mode of preparation of the CQDs. ${ }^{17,18}$ The highly emitting CQDs have been increasingly utilized in bioimaging $^{19-21}$ as well as in designing light emitting diodes (LEDs). ${ }^{22}$ Among other important applications, CQDs have been coupled with materials like $\mathrm{TiO}_{2}$ and $\mathrm{RuO}_{2}$ for applications in supercapacitors and efficient electrodes for batteries. ${ }^{23-26}$ 
Recently, we have reported conducting PSS-CQDs which were prepared in a single step pyrolysis of citric acid and poly(sodium 4-styrene sulfonate) (PSS) mixture. The PSS-CQDS showed stable redox behavior within an appreciable voltage regime. ${ }^{27}$ In the present manuscript, we have used a step-wise surface modification technique to convert powdered PSS-CQDS into CQD-NIMs at room temperature. The CQD-NIMs consisted of redox active CQDs ${ }^{27}$ as the core and Jeffamine ${ }^{\circledR}$ as the canopy (Scheme 1). The redox active CQD-NIM sample was used as an electrolyte and its CV properties were studied. Interestingly, it was observed that the extrinsic PL maxima $\left(\lambda_{\mathrm{em}}\right)$ of CQD-NIMs dynamically shift during and after CV cycles. The phenomenon was studied in detail and reasons for the dynamic $\lambda_{\mathrm{em}}$ behavior under CV induced polarization were predicted. The work may provide a PL-based approach towards understanding molecular canopy dynamics in NIM-like fluids. The use of redox-activeCQDs has been showcased as an effective core material in the NIM-class of materials that can be used in future electrochemical applications.

\section{Experimental}

\section{Materials}

Citric acid (99.7\%) was purchased from Himedia Chemicals. Poly(sodium 4-styrene sulfonate) (99.8\%, M.W. 70 000) (PSS) was purchased from Sigma-Aldrich and it was used as received without further purification. Jeffamine ${ }^{\circledR}$ (M2070) was obtained as a gifted sample from Huntsman (India). DOWEX ${ }^{\circledR}$ HCR-W2-hydrogen resin was purchased from Sigma-Aldrich. Dialysis membranes were purchased from Himedia. Milli-Q water was used in all experiments. All the measurements were performed at room temperature (ca. $25^{\circ} \mathrm{C}$ ) unless otherwise mentioned.

\section{Method of preparation of CQD-NIMs}

PSS-passivated CQDs (PSS-CQD) were prepared in a one-step pyrolysis method using citric acid and PSS as precursors. The process was adopted from our previously reported work. ${ }^{27}$

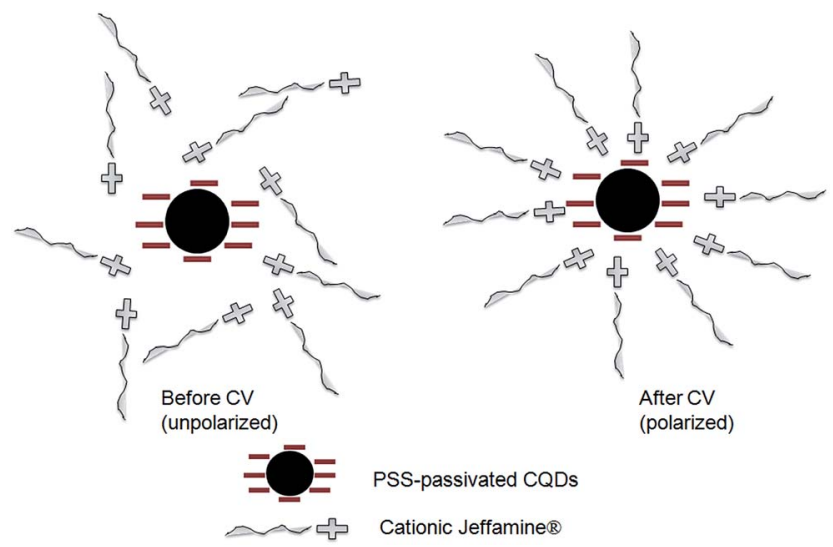

Scheme 1 Schematic representation of ordered and disordered structures of CQD-NIMs after and before CV.

\section{Ion exchange and surface modification of CQDS}

The ion exchange process to modify CQDs was done with slight modifications following our earlier reported method on the designing of gold nanorod-based NIMs. ${ }^{3}$ We have performed ion exchange in CQDs and modification of the ion exchanged product was done with Jeffamine ${ }^{\circledR}$. A column was prepared with Dowex HCR-W2 hydrogen resin. Before charging in the column, the resin was washed several times with Milli-Q water. For the preparation of CQD-NIMs, $0.5 \mathrm{~g}$ of PSS-CQD was dispersed in $150 \mathrm{ml}$ of Milli-Q water. The suspension was dialyzed with a HIMEDIA-110 dialysis membrane in Milli-Q water for $24 \mathrm{~h}$ and passed through the resin-packed column. The ion-exchange process was repeated three times to ensure that $\mathrm{Na}^{+}$ions present in CQDs get completely replaced by $\mathrm{H}^{+}$ions. The eluate showed a $\mathrm{pH}$ value of 2.6. It was collected from the column and titrated with $0.1 \mathrm{M}$ aqueous Jeffamine ${ }^{\circledR}$ solution. The final $\mathrm{pH}$ of the suspension was recorded to be 3.5. Similarly two other CQD-NIMs were prepared at different $\mathrm{pHs}(\mathrm{pH} 4.5$ \& 5.1). The Jeffamine ${ }^{\circledR}$ content increases with increase in $\mathrm{pH}$. The suspension was freeze-dried and CQD-NIMs were obtained as a brown liquid. The liquid was further dried and degassed in a vacuum desiccator. The final products were solvent free and liquid at room temperature. CV studies indicated that CQDNIMs prepared at $\mathrm{pH} 3.5$ show redox properties.

\section{Equipment and characterization techniques}

Dynamic Light Scattering (DLS) was performed with a Zetasizer from Malvern. Thermal degradation of CQD-NIMs was studied by thermogravimetry (TGA) using a NETZSCH STA 449 F3 Jupiter thermal analyzer. PL data were recorded using a Shimadzu RF5301 Fluorimeter. All the steady-state measurements were carried out using a HORIBA Jobin Yvon Fluorimax-4 Fluorimeter. PL spectra of CQD-NIMs were recorded upon excitation at $420 \mathrm{~nm}$ using a slit width of $3 \mathrm{~nm}$. The corresponding emission maxima $\left(\lambda_{\mathrm{em}}\right)$ were recorded in the regime of 450-500 $\mathrm{nm}$. Life-time studies indicated that the emission band of $\sim 500 \mathrm{~nm}$ has a life-time of $\sim 3.9 \mathrm{~ns}$ as shown in the ESI (Fig. ESI $1 \dagger$ ). This band must correspond to the $\lambda_{\text {em }}$ as reported by Wen et al. ${ }^{14} \lambda_{\text {em }}$ with a large bandwidth originates from the surface states present in CQDs and which is well documented in the literature. During CV cycles, the surface of the CQDs is polarized due to changes in the polarity of the surface environment, i.e., changes in the conformation of the Jeffamine ${ }^{\circledR}$ canopy. Hence to understand the phenomenon with fluorescence, we decided to measure the $\lambda_{\mathrm{em}}$. The $\lambda_{\mathrm{em}}$ was recorded immediately before and after the CV experiment with solid state accessories. The CQD-NIMs fluid was collected from the CV workstation and drop coated on a spectroscopic grade quartz plate. The same was placed inside the solid-state accessory of the spectrofluorometer. A differential scanning calorimetry (DSC) device (Pyris Diamond DSC, Perkin-Elmer) was used to study the glass-transition temperatures $\left(T_{\mathrm{g}}\right)$ of the CQD-NIMs. The samples were kept at $50{ }^{\circ} \mathrm{C}$ for $30 \mathrm{~min}$ under isothermal conditions and scanned from $50{ }^{\circ} \mathrm{C}$ to $100{ }^{\circ} \mathrm{C}$ at a heating rate of $10{ }^{\circ} \mathrm{C} \min ^{-1}$, then cooled to $-100{ }^{\circ} \mathrm{C}$. Two cycles were 
performed. The DSC equipment was calibrated using In and Zn as calibration materials prior to scanning the samples.

\section{Cyclic voltammetry}

$\mathrm{CV}$ experiments were performed by using $\mathrm{CH}$ instruments ( $\mathrm{CHI}$ 600E) Austin, Texas. Before electrochemical measurements at the metallic electrode, the CQD-NIMs fluid was degassed and dried under vacuum for a few days so that no traces of water or oxygen remain within the fluid. For electrochemical measurements at a Pt electrode, CQD-NIMs were transferred to an air tight glass cell $(\varphi 25 \mathrm{~mm} \times 40 \mathrm{~mm})$ with a Teflon cap $(\varphi 30 \mathrm{~mm})$. A three electrode system was used where Pt was taken as the working electrode (metal disk encapsulated by Kel-F $\varphi 6.35 \mathrm{~mm}$ ) and Pt-wire $(\varphi 0.5 \mathrm{~mm})$ and $\mathrm{Ag} / \mathrm{AgCl}$ were used as the counter and reference electrodes, respectively.

\section{Results and discussions}

The CQD-NIMs were designed by a resin-exchange process followed by a simple acid-base titration as detailed in the experimental section. ${ }^{3}$ During titration, the surface $-\mathrm{SO}_{3} \mathrm{H}$ groups on CQDs exchange the proton with the terminal amine group of Jeffamine ${ }^{\circledR}$ creating an anionic corona and a cationic canopy as shown in Scheme 1. ${ }^{3}$ DLS of CQD-NIMs was obtained after dialyzing the samples with the HIMEDIA-110 dialysis membrane followed by filtration with a $0.22 \mu \mathrm{m}$ cellulose nitrate membrane. The intensity average hydrodynamic radius of CQDNIMs shows two peaks at $\sim 10.5 \mathrm{~nm}$ and $32.8 \mathrm{~nm}$, respectively (Fig. 1A). The first peak $(10.5 \mathrm{~nm})$ is due to individual CQDs and is in agreement with the size of CQDs observed in TEM images. ${ }^{27}$ The second peak at $32.8 \mathrm{~nm}$ may be due to the canopy formation by Jeffamine ${ }^{\circledR}$ around PSS-CQDs. Multiple particles can also form cores leading to different sizes of core-coronacanopy structures and thus multiple peaks may be detected. ${ }^{3} \mathrm{~A}$ lower negative zeta potential value $(-10.4 \mathrm{mV})$ of CQD-NIMs (Fig. 1B) together with a tailing towards the positive zeta region indicate surface charge neutralization in the presence of cationic Jeffamine ${ }^{\circledR}$ on the PSS-CQDs surface. From DLS it is apparent that there is a core CQD which might be surrounded by a polymer canopy as shown in Scheme 1 .

The CQD-NIMs showed enhanced solubility in a wide range of solvents as shown in Fig. ESI2. $\dagger$ The enhanced solubility can be attributed to the amphiphilic nature of Jeffamine ${ }^{\circledR}$ as the canopy. Similar observations have been reported for fullerol and graphene-oxide based NIMs. ${ }^{4,5}$ The presence of Jeffamine ${ }^{\circledR}$ was evident in the TGA thermogram. TGA of CQD-NIMs (Fig. 1C) shows a $96.6 \%$ mass loss at $\sim 407{ }^{\circ} \mathrm{C}$ which is due to degradation of the Jeffamine ${ }^{\circledR}$ canopy attached to the core CQDs through electrostatic interactions with the PSS corona. At around $300{ }^{\circ} \mathrm{C}$, a small change in the mass was observed which might be due to degradation of PSS attached with CQDS $(\sim 3 \%) .{ }^{27}$ CQD-NIMs have a fluid-like appearance at room
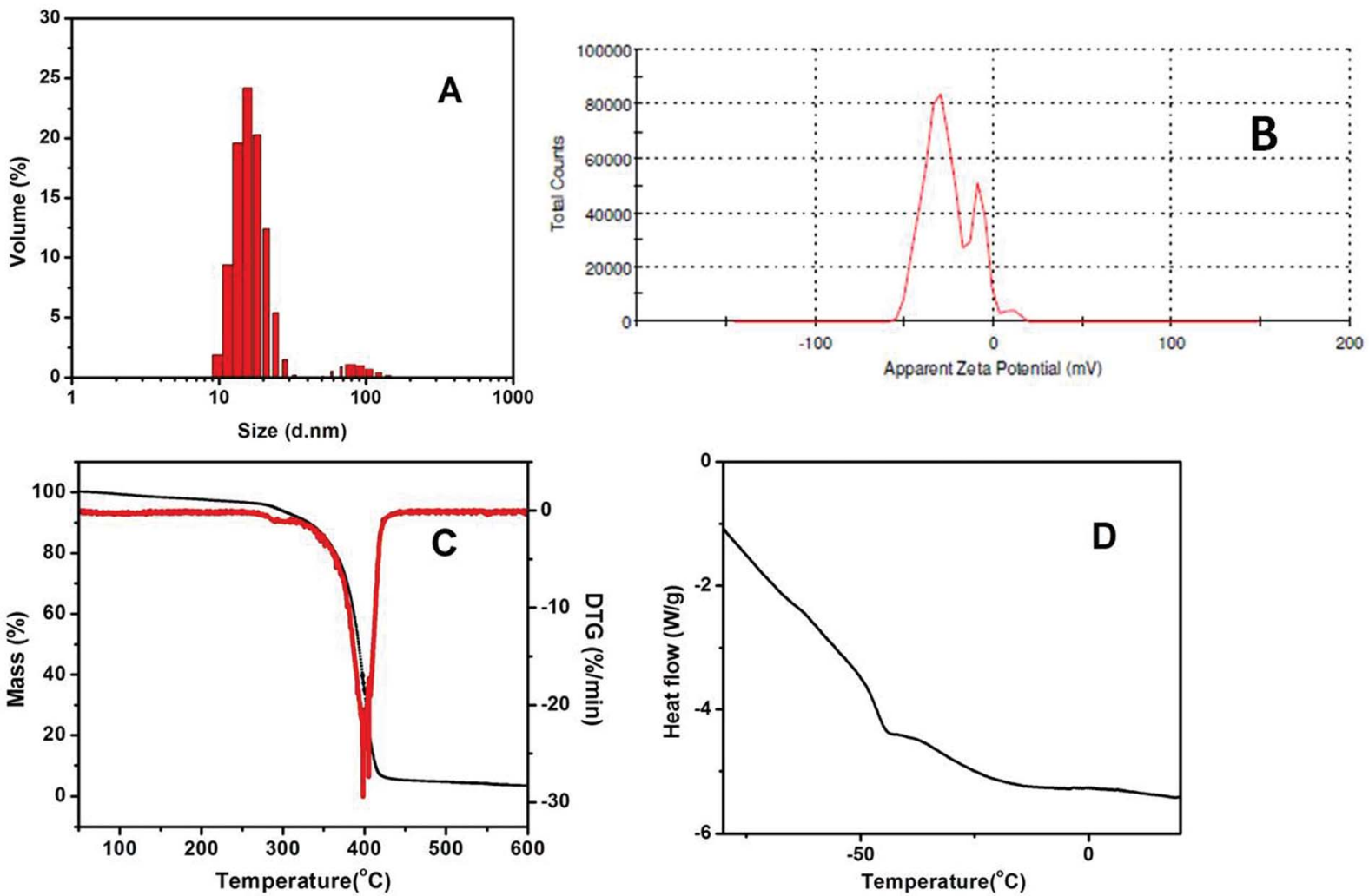

Fig. 1 (A) DLS data of CQD-NIM volume-size distribution, (B) surface charge measurement through the zeta potential, (C) thermal stability (red and black correspond to DTG \& TGA, respectively) and (D) DSC data for glass transition temperature of CQD-NIMS. 
temperature. The DSC thermogram (Fig. 1D) shows that the $T_{\mathrm{g}}$ of the CQD-NIMs is $\sim-50{ }^{\circ} \mathrm{C}$. This value is lower than the room temperature and hence the material is a liquid at room temperature. The $T_{\mathrm{g}}$ value of pure Jeffamine ${ }^{\circledR}$ is $\sim-71^{\circ} \mathrm{C} .{ }^{4}$ The increase in the $T_{\mathrm{g}}$ value of CQD-NIMs compared to that of pure polymer indicates the formation of a core-canopy structure. A similar observation has been reported for fullerol-based NIMs. ${ }^{3}$

The CQD-NIMs fluid was taken as the electrolyte for the CV experiment and no additional electrolyte was added. The starting potential was $-3.0 \mathrm{~V}$. It was scanned till the switch potential point of $+3.0 \mathrm{~V}$ and swiped back to the end potential at $-3.0 \mathrm{~V}$. The fluid was stable in the voltage regime of $6 \mathrm{~V}$. The scan rate was kept fixed at $50 \mathrm{mV} \mathrm{s}^{-1}$. PL spectra recorded upon excitation at $420 \mathrm{~nm}$ before and after the CV experiments, showed a red-shift in $\lambda_{\text {em }}$ (inset in Fig. 2). The PL spectra of CQD-NIMs recorded before CV show $\lambda_{\text {em }}$ at $494 \mathrm{~nm}$, that redshifted to $506 \mathrm{~nm}$ after $5 \mathrm{CV}$ cycles (inset in Fig. 2). Different numbers of $\mathrm{CV}$ cycles were performed and $\lambda_{\mathrm{em}}$ was recorded immediately after the completion of each run. The $\lambda_{\mathrm{em}}$ was plotted with the number of cycles as shown in Fig. 2 . The plot indicated no red-shift beyond 5 cycles. Interestingly, $\lambda_{\mathrm{em}}$ was observed to gradually blue-shift with time as is evident from time dependent studies performed after $10 \mathrm{CV}$ cycles (Fig. 3). After $\sim 2 \mathrm{~h}$, the $\lambda_{\text {em }}$ was observed at $494 \mathrm{~nm}$, which was the original $\lambda_{\mathrm{em}}$ before $\mathrm{CV}$.

Spectral changes observed for $\lambda_{\text {em }}$ (Fig. $2 \& 3$ ) of CQD-NIMs can be related to the dynamic changes in the surface environment under polarization. Initially, the Jeffamine ${ }^{\circledR}$ matrix embedding the CQDs remain un-polarized (Scheme 1). The free electrons of CQDs populate the surface trap states (STS) at room temperature. ${ }^{27}$ During the forward scan in CV (oxidation), the electron transfers from the STS of CQDs to the +ve electrode as shown in Scheme 2. In the reverse scan (reduction), the electron transfers from the -ve electrode to the LUMO of the CQDs. After $\mathrm{CV}$ (relaxation), electrons from the LUMO can get transferred to the Jeffamine® canopy through STS as shown in Scheme 2. This is possible as the Jeffamine ${ }^{\circledR}$ canopy in the CQD-NIMs is cationic and hence electron deficient. Electron donation to the

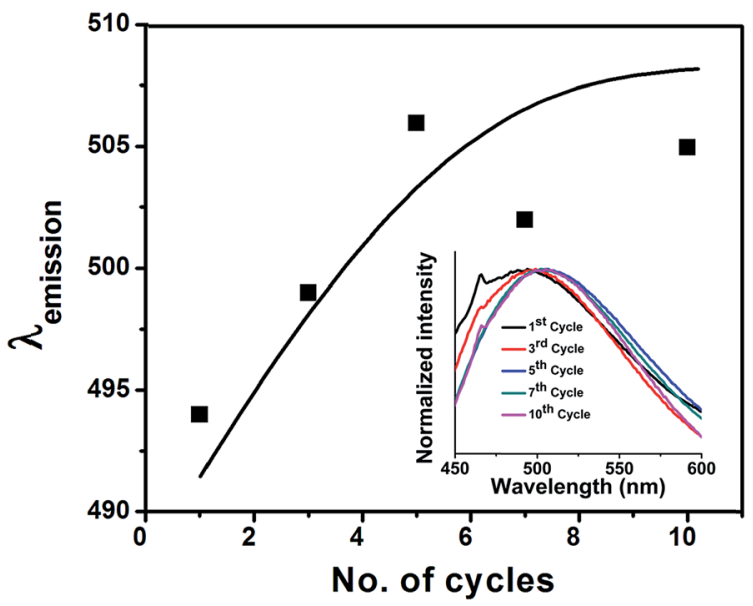

Fig. 2 Plot of $\lambda_{\text {em }}$ of CQD-NIMs against the number of CV cycles. The inset shows the PL spectra as a function of CV cycles.

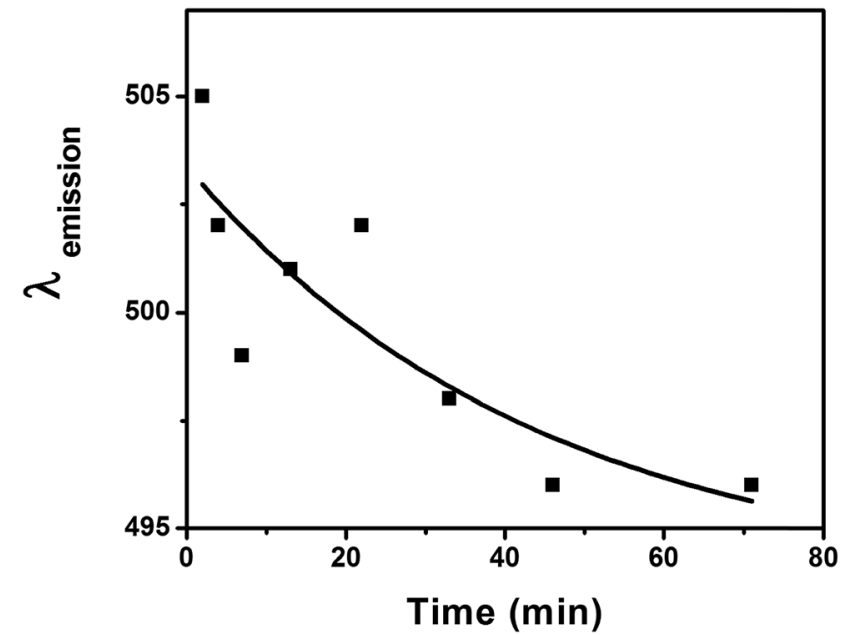

Fig. 3 Plot of $\lambda_{\text {em }}$ of CQD-NIMs recorded after $10 \mathrm{CV}$ cycles against relaxation time.

Jeffamine ${ }^{\circledR}$ canopy partially polarizes the CQD-NIMs structure thus creating a compact canopy (Scheme 2). Formation of a compact cationic Jeffamine ${ }^{\circledR}$ canopy around the anionic CQD passivated PSS core-corona structure can lower the energy of the STS (Scheme 2). The situation is similar to the lowering of the excited energy state of a molecule due to changes in the polarity of solvent molecules in solution. ${ }^{28}$ The lowering in energy of the STS is evident in the red-shift of $\lambda_{\text {em }}$ of the CQDS after CV cycles (Fig. 2). The extent of polarization of the canopy increases with increase in the number of CV cycles. Such polarization strengthens the cationic Jeffamine ${ }^{\circledR}$ canopy resulting in further stabilization of the STS present on the core CQDs (Scheme 2). The stabilization leads to a gradual red shift in $\lambda_{\text {em }}$ as indicated in Fig. 2 . $\lambda_{\text {em }}$ values acquire a limiting value after 5 cycles indicating attainment of maximum polarization and absolute compactness of the corona-canopy structure as shown in Scheme 2. The relaxation dynamics of the coronacanopy structure of CQD-NIMs can be visualized through the time dependent blue-shift of $\lambda_{\mathrm{em}}$ as shown in Fig. 3. Considering that the cationic Jeffamine ${ }^{\circledR}$ canopy is dynamic and it moves from one anionic CQD to another, we can predict that the polarized structure of the Jeffamine ${ }^{\circledR}$ canopy relaxes once $\mathrm{CV}$ is turned off. ${ }^{6}$ The relaxation is driven by the laws of thermodynamics. During polarization, the ordering of the canopy around the CQD corona decreases the entropy of the system. In order to gain entropy, that is to create the random/un-polarized state, the systems relax back to their original conformation (Scheme 1). The randomness in the Jeffamine ${ }^{\circledR}$ canopy structure removes its stabilizing effect on the surface trap state of CQDs. Consecutively, the energy of the surface trap states of CQDs increases as indicated by the gradual blue-shift in extrinsic $\lambda_{\mathrm{em}}$ values (Fig. 3). The changes in the energy of the surface trap states due to dynamic changes in the surface environment of

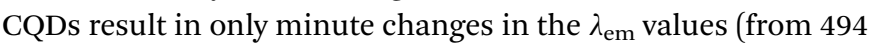
to $505 \mathrm{~nm}$ ). The shifts in $\lambda_{\text {em }}$ values with minute changes in the polarity in the immediate vicinity of the CQD surface can be observed upon exposure of the CQD-NIMs to different solvents. 


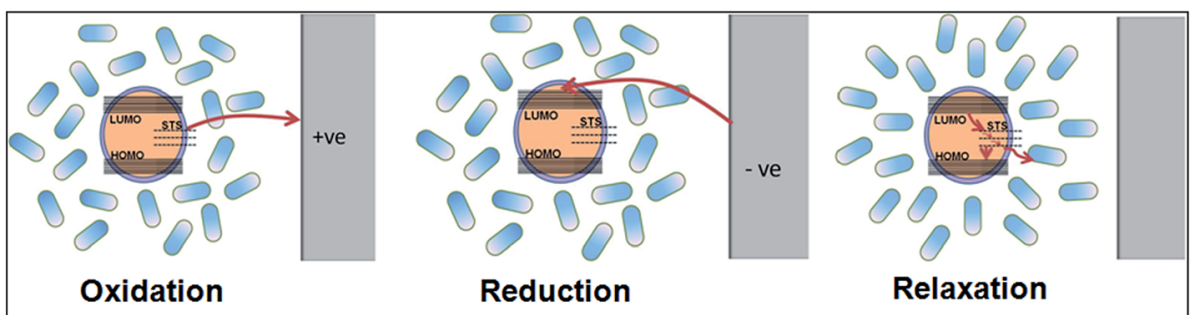

Scheme 2 Schematic representation of CV induced polarization of CQD-NIMs. The diagram shows different stages of electron transfer during the CV cycle.

Thus, the $\lambda_{\mathrm{em}}$ values for water and ethanol were observed at 445 and $455 \mathrm{~nm}$, respectively. The effect of the solvent dielectric constant on $\lambda_{\mathrm{em}}$ is well reported in the literature. ${ }^{28}$

$\mathrm{CV}$ data obtained from the first cycle shows the oxidation and reduction peaks at $-0.83 \mathrm{~V}$ and $+1.0 \mathrm{~V}$, respectively (Fig. 4). The peaks appear due to the redox process of the CQDs. ${ }^{27}$ Such a saturating nature of the $\mathrm{CV}$ curve indicates that the to \& fro electron transfer process between CQDs and electrodes is quite fast. It is also noticed that unlike the $\mathrm{CV}$ of aqueous CQD, ${ }^{27}$ the current here constantly increases with the potential, making a "paunch" or a window in the CV spectra (Fig. 4). This can be attributed to the interactions of negatively charged CQDs with the cationic Jeffamine ${ }^{\circledR}$ canopy through electrostatic interactions. ${ }^{29,30}$ Due to this interaction and surrounding insulating Jeffamine ${ }^{\circledR}$ matrix, there is a consistent flow of charges through the electrolyte (CQD-NIMs). Nevertheless, within this paunch area i.e. from $-1.0 \mathrm{~V}$ to $+2.5 \mathrm{~V}$, which has been created due to the reduction of CQDs, the material can pile up enough charge carriers. ${ }^{31} \mathrm{CV}$ characterization of only Jeffamine ${ }^{\circledR}$ shows no significant redox properties (Fig. ESI3†). CQD-NIM samples prepared at higher Jeffamine ${ }^{\circledR}$ contents did not show redox behavior. This may be due to greater concentrations of insulating Jeffamine ${ }^{\circledR}$ present in those systems.

Interestingly, though $\mathrm{CV}$ experiments revealed the presence of the oxidation peak at $1.0 \mathrm{~V}$ during the first scan, further cycles showed oxidation at $1.4 \mathrm{~V}$ that deviate slightly in further cycles

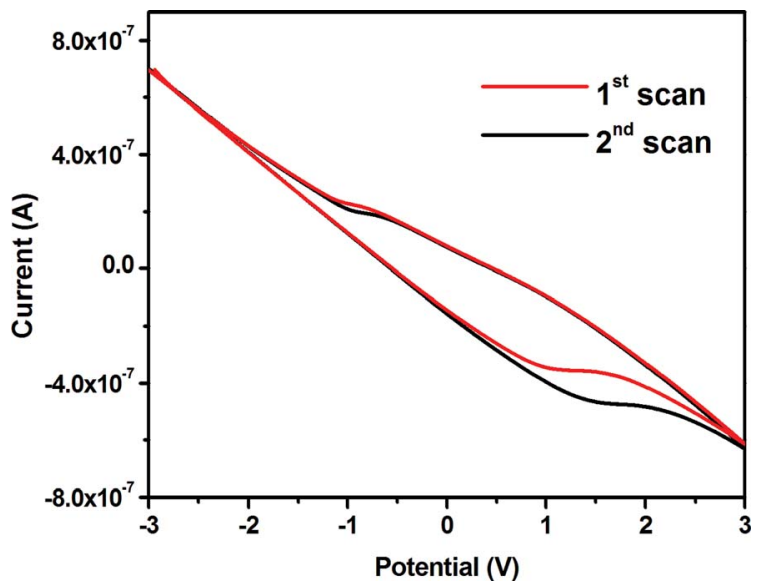

Fig. 4 CV of CQD-NIMs showing the first \& second cycles, respectively.
(Fig. 4 \& ESI4 $\dagger$ ). When CV was run after a delay, the first scan showed the oxidation peak position to be back at $1.0 \mathrm{~V}$. Such an increase in the oxidation potential is related to the stabilization of surface trap states in CQDs during polarization of the Jeffamine ${ }^{\circledR}$ canopy. ${ }^{7,13}$ During the forward scan in CV (oxidation), Jeffamine ${ }^{\circledR}$ polarizes near the CQD surface (Scheme 2). The compact canopy results in stabilization of the surface trap states in CQDs. ${ }^{32,33}$ A decrease in the energy level creates a hindrance for electron transfer from CQDs to the +ve electrode surface in the consecutive $\mathrm{CV}$ cycles. Hence, the increase in the oxidation potential after the first cycle (1 to $1.4 \mathrm{~V}$ ) was observed (Fig. 4). In consecutive cycles, the canopy structure is further reinforced which results in a slightly higher energy shift of the oxidation peak in consecutive CV cycles as shown in Fig. ESI4. $\dagger$ After 10 cycles, there was no further shift in the oxidation peak position (Fig. ESI $4 \dagger$ ). Once the CV is switched off and the CQDNIMs were allowed to relax, the original surface energy states are repopulated (peak at a lower voltage of $1.0 \mathrm{~V}$ is observed). ${ }^{30}$ The slight increase in the life-time of the CQD-NIMs after different CV cycles (Fig. ESI1 $\dagger$ ) indicates a decrease in the radiative decay rate due to stabilization of surface trap states as a function of polarization. The same is in line with the red-shift of extrinsic emission maxima with increasing $\mathrm{CV}$ cycles as observed in the manuscript. Thus both PL and CV results reinforce the fact that dynamic changes in the canopy structure under polarization influence surface trap states of core CQDs in the CQD-NIM fluid.

In conclusion, we have shown that the extrinsic PL band $\left(\lambda_{\mathrm{em}}\right)$ of CQD-NIMs shows spontaneous and reversible fluctuations (blue shift \& red shift) under polarization. Though the red shift is only $12 \mathrm{~nm}$ (494 to $506 \mathrm{~nm}$ after 10 consecutive CV cycles), it is of immense importance. There was neither any change in solvent polarity nor any ligand exchange processes on the surface of CQDs. The red-shift can only be interpreted by considering, ordering and disordering of the Jeffamine ${ }^{\circledR}$ canopy in the immediate vicinity of the CQD surface in the NIM material. The canopy dynamics influenced fluctuations in the surface trap states of CQDs. This was proved from the CV data of CQD-NIMs obtained from multiple cycles. Finally, $\lambda_{\mathrm{em}}$ was shown to be used for monitoring the molecular canopy dynamics of NIMs. The simplicity of the method allows realization of the canopy dynamics through the PL approach and can be helpful to prepare the green electrolyte material for future battery applications. 


\section{Acknowledgements}

This work was funded by the Department of Science \& Technology (New Delhi) (SR/FT/CS-128/2010). LB thanks the Ministry of Earth Sciences (MoES/P.O.(Seismo)/1(190)/2013) for support and fellowship. Support from PSG Institute of Advanced Studies is gratefully acknowledged. We thank Prof. Tushar Jana (Central University of Hyderabad, India) for DSC experiments. Finally we thank HUNTSMAN (India) for providing the Jeffamine® polymer as the gift sample.

\section{References}

1 R. Rodriguez, R. Herrera, L. A. Archer and E. P. Giannelis, Adv. Mater., 2008, 20, 4353.

2 A. B. Bourlinos, S. R. Chowdhury, R. Herrera, D. D. Jiang, Q. Zhang, L. A. Archer and E. P. Giannelis, Adv. Funct. Mater., 2005, 15, 1285.

3 R. R. Bhattacharjee, R. Li, L. Estevez, D.-M. Smilgies, A. Amassian and E. P. Giannelis, J. Mater. Chem., 2009, 19, 8728.

4 N. Fernandes, P. Dallas, R. Rodriguez, A. B. Bourlinos, V. Georgakilas and E. P. Giannelis, Nanoscale, 2010, 2, 1653. 5 C. Zeng, Z. Tang, B. Guo and L. Zhang, Phys. Chem. Chem. Phys., 2012, 14, 9838.

6 M. L. Jespersen, P. A. Mirau, E. von Meerwall, R. A. Vaia, R. Rodriguez and E. P. Giannelis, Nanoscale, 2010, 4, 3735.

7 A. B. Bourlinos, A. Stassinopoulos, D. Anglos, R. Zboril, M. Karakassides and E. P. Giannelis, Small, 2008, 4, 455.

8 J. Wang, C. F. Wang and S. Chen, Angew. Chem., Int. Ed., 2012, 51, 1.

9 Y.-P. Sun, B. Zhou, Y. Lin, W. Wang, K. A. S. Fernando, P. Pathak, B. A. Harruff, X. Wang, H. Wang, P. G. Luo, H. Yang, B. Chen, L. M. Veca and S.-Y. Xie, J. Am. Chem. Soc., 2006, 128, 7756.

10 L. Cao, X. Wang, M. J. Meziani, F. Lu, H. Wang, P. G. Luo, Y. Lin, B. A. Harruff, L. M. Veca, D. Murray, S.-Y. Xie and Y.-P. Sun, J. Am. Chem. Soc., 2007, 129, 11318.

11 F. Liu, M.-H. Jang, H. D. Ha, J.-H. Kim, Y.-H. Cho and T. S. Seo, Adv. Mater., 2013, 25, 3657.

12 P. Yu, X. Wen, Y.-R. Toh and J. Tang, J. Phys. Chem. C, 2012, 116, 25552.
13 L. Cao, M. J. Meziani, S. Sahu and Y. P. Sun, Acc. Chem. Res., 2013, 46, 171.

14 X. Wen, P. Yu, Y.-R. Toh, X. Hao and J. Tang, Adv. Opt. Mater., 2013, 1, 173.

15 S. Mondal, U. Rana and S. Malik, Chem. Commun., 2015, 51, 12365.

16 J. Wang, C. F. Wang and S. Chen, Angew. Chem., Int. Ed., 2012, 51, 1.

17 Y. Wang and A. Hu, J. Mater. Chem. C, 2014, 2, 6921.

18 H. Li, Z. Kang, Y. Liu and S.-T. Lee, J. Mater. Chem., 2012, 22, 24230.

19 S. C. Ray, A. Saha, N. R. Jana and R. Sarkar, J. Phys. Chem. C, 2009, 113, 18546.

20 L. Cao, S. T. Yang, X. Wang, P. G. Luo, J. H. Liu and S. Sahu, Theranostics, 2012, 2, 295.

21 M. Nurunnabi, Z. Khatun, K. M. Huh, S. Y. Park, Y. D. Lee, K. J. Cho, et al., ACS Nano, 2013, 7, 6858.

22 S. Y. Lim, W. Shen and Z. Gao, Chem. Soc. Rev., 2015, 44, 362.

23 X. Chen, Q. Jin, L. Wu, C. Tung and X. Tang, Angew. Chem., Int. Ed., 2014, 53, 12542.

24 Y. Zhu, X. Ji, C. Pan, Q. Sun, W. Song, L. Fang, Q. Chen and C. E. Banks, Energy Environ. Sci., 2013, 6, 3665.

25 D. Chao, C. Zhu, X. Xia, J. Liu, X. Zhang, J. Wang, P. Liang, J. Lin, H. Zhang, Z. Xiang Shen and H. J. Fan, Nano Lett., 2015, 15, 565.

26 Y. Yang, X. Ji, M. Jing, H. Hou, Y. Zhu, L. Fang, X. Yang, Q. Chen and C. E. Banks, J. Mater. Chem. A, 2015, 3, 5648.

27 L. Bhattacharjee, R. Manoharan, K. Mohanta and R. R. Bhattacharjee, J. Mater. Chem. A, 2015, 3, 1580.

28 J. R. Lakowicz, Principles of Fluorescence Spectroscopy, Plenum Press, New York, London, 1st edn, 1983.

29 Electroanalytical Method-Guide to Experiments and Applications, ed. F. Scholz, Springer, 2002, DOI: 10.1007/ 978-3-662-04757-6.

30 K. Morinaga, Bull. Chem. Soc. Jpn., 1956, 29, 793.

31 H. Wang, A. Thiele and L. Pilon, J. Phys. Chem. C, 2013, 117, 18286.

32 J. H. Engel, Y. Surendranath and A. Paul Alivisatos, J. Am. Chem. Soc., 2012, 134, 13200.

33 M. Jankulovska, T. Berger, S. S. Wong, R. Gómez and T. LanaVillarreal, ChemPhysChem, 2012, 13, 3008. 